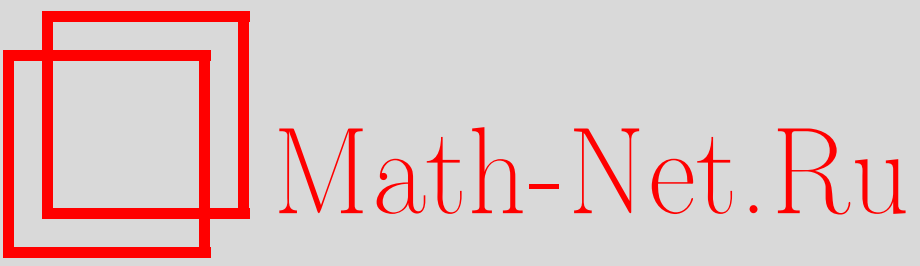

А. А. Битюрин, В. К. Манжосов, Продольный удар о жёсткую преграду однородного и ступенчатого стержней при понижении продольной жёсткости, Вестн. Сам. гос. техн. ун-та. Сер. Физ.-мат. науки, 2010, выпуск 5(), 285-290

DOI: https://doi.org/10.14498/vsgtu725

Использование Общероссийского математического портала Math-Net.Ru подразумевает, что вы прочитали и согласны с пользовательским соглашением

http: //www.mathnet.ru/rus/agreement

Параметры загрузки:

IP : 54.198 .64 .247

26 апреля 2023 г., 12:12:35 
УДК 517.958+622.233.6

\section{ПРОДОЛЬНЫЙ УДАР О ЖЁСТКУЮ ПРЕГРАДУ ОДНОРОДНОГО И СТУПЕНЧАТОГО СТЕРЖНЕЙ ПРИ ПОНИЖЕНИИ ПРОДОЛЬНОЙ ЖЁСТКОСТИ}

\section{А. А. Битюрин, В. К. Манжосов}

Ульяновский государственный технический университет, 432027, Ульяновск, ул. Северный Венец, 32.

E-mail: Denjgy0706@yandex.ru

Рассматривается продольный упругий центральный удар неоднородной стержневой системы о жёсткую преграду при неудерживаюших связях путём аналитического решения волнового дифференииального уравнения методом Даламбера. Стержсневая система состоит из однородного стержня постоянного поперечного сечения и ступенчатого неоднородного стержня, имеющего два однородных участка различной длины и площади поперечных сечений. Связи с жёсткой преградой и между стержнями неудерживающие.

Ключевые слова: удар, дебормация, математическое моделирование, скоростъ.

1. Введение. В основе технологических операций с использованием машин ударного действия лежит нанесение продольного удара неоднородного многоступенчатого упругого стержня или системы стержней.

Особый интерес представляет рассмотрение процесса удара рабочим инструментом по абсолютно жёсткой преграде, поскольку данная ситуация в технологическом процессе является аварийной. Очень важно, чтобы ударная машина имела минимальные повреждения и сохраняла свою работоспособность. В случае выполнения данного условия машина будет работать абсолютно надёжно при обработке технологической среды любой жёсткости. Следовательно, именно здесь наиболее остро встаёт вопрос правильного подбора рабочих элементов ударной машины.

2. Постановка задачи. В известных работах модель учёта неудерживающих связей в задачах продольного удара стержней сводится к тому, что процесс удара считался завершённым, если в ударном сечении возникла деформация растяжения и происходил разрыв связи. Возможность повторного соударения стержней исследователями не рассматривалась. Такая модель продольного удара, с одной стороны, отсекала информацию о последующем нагружении стержня при повторных соударениях, а с другой стороны, представляла некорректную информацию о восстановлении скорости стержня при продольном ударе.

В данной работе представлена математическая модель продольного удара стержней при разрывах связей и возникновении повторных соударений [1-6].

Рассмотрена модель продольного удара о жёсткую преграду системы однородного стержня постоянного поперечного сечения и неоднородного ступенчатого стержня (рис. 1). Масса однородного стержня $m_{1}$, длина $l_{1}$. Начальный и конечный участки ступенчатого стержня имеют длины соответственно $l_{2}$ и $l_{3}$, масса стержня $m_{2}$. Общая длина обоих стержней равна $l$. Предударная скорость стержневой системы равна $V_{0}$. Все участки состоят из одного материала.

Движение поперечных сечений соударяемых стержней описывается волновым

Анатолий Александрович Битюрин (к.т.н., доцент), доцент, каф. теоретической и прикладной механики. Владимир Кузъмич Манжосов (д.т.н., профессор), зав. кафедрой, каф. теоретической и прикладной механики. 


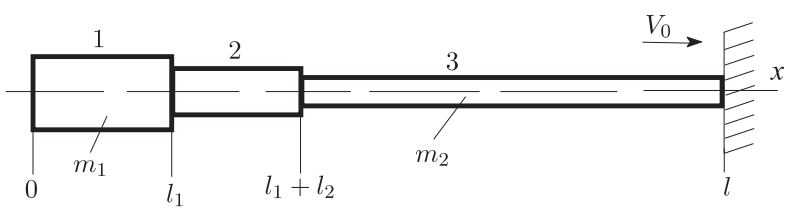

Рис. 1. Схема удара неоднородной стержневой системы о жёсткую преграду при неудерживающих связях: 1 - однородный стержень; 2 - начальный участок ступенчатого стержня; 3 конечный участок ступенчатого стержня

дифференциальным уравнением

$$
\begin{gathered}
\frac{\partial^{2} u_{i}}{\partial x^{2}}-\frac{1}{a^{2}} \frac{\partial^{2} u_{i}}{\partial t^{2}}=0 \\
\text { при } i=1, x \in\left[0, l_{1}\right] ; \quad i=2, x \in\left[l_{1}, l_{1}+l_{2}\right] ; \quad i=3, x \in\left[l_{1}+l_{2}, l\right],
\end{gathered}
$$

где $u_{i}(x, t)$ - продольное перемещение поперечного сечения соответственно однородного стержня 1 , начального 2 и конечного 3 участков неоднородного стержня, $x-$ координата сечения, $t$ - время, $a$ - скорость распространения продольной волны деформации.

Начальные условия определяют состояние участков стержневой системы перед их соударением при $t=t_{0}=0$ :

$$
\begin{array}{rlrl}
\frac{\partial u_{1}\left(x, t_{0}\right)}{\partial t}=V_{0}, & \frac{\partial u_{1}\left(x, t_{0}\right)}{\partial x}=0, & \frac{\partial u_{2}\left(x, t_{0}\right)}{\partial t}=V_{0}, & \frac{\partial u_{2}\left(x, t_{0}\right)}{\partial x}=0, \\
\frac{\partial u_{3}\left(x, t_{0}\right)}{\partial t}=V_{0}, & \frac{\partial u_{3}\left(x, t_{0}\right)}{\partial x}=0 . &
\end{array}
$$

Краевые условия определяют отсутствие силы в сечении $x=0$ и равенство нулю скорости сечения $x=l$ при взаимодействии ступенчатого стержня с жёсткой преградой:

$$
\frac{\partial u_{1}(0, t)}{\partial x}=0, \quad \frac{\partial u_{3}(l, t)}{\partial t}=0, \quad \text { если } \quad \frac{\partial u_{3}(l, t)}{\partial x}<0,
$$

а также равенство сил и скоростей в контактных сечениях $x=l_{1}$ однородного стержня и начального участка ступенчатого стержня при непосредственном их взаимодействии:

$$
\begin{aligned}
E A_{1} \frac{\partial u_{1}\left(l_{1}, t\right)}{\partial x} & =E A_{2} \frac{\partial u_{2}\left(l_{1}, t\right)}{\partial x}, \quad \text { если } \frac{\partial u_{1}\left(l_{1}, t\right)}{\partial x}<0, \\
\frac{\partial u_{1}\left(l_{1}, t\right)}{\partial t} & =\frac{\partial u_{2}\left(l_{1}, t\right)}{\partial t}, \quad \text { если } \quad \frac{\partial u_{1}\left(l_{1}, t\right)}{\partial x}<0,
\end{aligned}
$$

либо отсутствие сил в ударных сечениях стержней, если их взаимодействие отсутствует:

$$
\frac{\partial u_{1}\left(l_{1}, t\right)}{\partial x}=0, \quad \frac{\partial u_{2}\left(l_{1}, t\right)}{\partial x}=0, \quad \text { если } \quad u_{1}\left(l_{1}, t\right)-u_{2}\left(l_{1}, t\right) \leqslant 0,
$$

где $E$ - модуль упругости первого рода, $A_{1}$ - площадь поперечного сечения однородного стержня, $A_{2}$ - площадь поперечного сечения участка 2 ступенчатого стержня (участок 2).

В переходном сечении $x=l_{1}+l_{2}$ начального и конечного участков ступенчатого стержня краевые условия также определяют равенство сил и скоростей:

$$
E A_{2} \frac{\partial u_{2}\left(l_{1}+l_{2}, t\right)}{\partial x}=E A_{3} \frac{\partial u_{3}\left(l_{1}+l_{2}, t\right)}{\partial x}
$$




$$
\frac{\partial u_{2}\left(l_{1}+l_{2}, t\right)}{\partial t}=\frac{\partial u_{3}\left(l_{1}+l_{2}, t\right)}{\partial t},
$$

где $A_{3}$ - площадь поперечного сечения участка 3 ступенчатого стержня.

Разрыв контакта в сечениях $x=l_{1}$ и $x=l$ происходит при условии отрицательной разности скоростей в сечениях $x=l_{1}$ однородного стержня и начального участка ступенчатого стержня (участка 2 стержневой системы), а также отрицательной скорости сечения $x=l$ конечного участка ступенчатого стержня:

$$
\frac{\partial u_{1}\left(l_{1}, t\right)}{\partial t}-\frac{\partial u_{2}\left(l_{1}, t\right)}{\partial t}<0, \quad \frac{\partial u_{3}(l, t)}{\partial t}<0 .
$$

Повторный удар в сечениях с неудерживающими связями может возникнуть после разрыва контакта в этих сечениях при условии положительной разности скоростей в сечениях $x=l_{1}$ однородного стержня (участка 1 стержневой системы) и начального участка ступенчатого стержня, а также положительной скорости сечения $x=l$ :

$$
\frac{\partial u_{1}\left(l_{1}, t\right)}{\partial t}-\frac{\partial u_{2}\left(l_{1}, t\right)}{\partial t}>0, \quad \frac{\partial u_{3}(l, t)}{\partial t}>0
$$

3. Метод решения. Решение дифференциального уравнения (1) реализуется методом Даламбера в виде суммы двух разрывных функций [1]:

$$
\begin{gathered}
u_{i}(x, t)=f_{i}(a t-x)+\varphi_{i}(a t+x) ; \\
\frac{\partial u_{i}(x, t)}{\partial x}=-f_{i}^{\prime}(a t-x)+\varphi_{i}^{\prime}(a t+x), \quad \frac{\partial u_{i}(x, t)}{\partial t}=a\left[f_{i}^{\prime}(a t-x)+\varphi_{1}^{\prime}(a t+x)\right] \\
\text { при } i=1, x \in\left[0, l_{1}\right] ; \quad i=2, x \in\left[l_{1}, l_{1}+l_{2}\right] ; \quad i=3, x \in\left[l_{1}+l_{2}, l\right],
\end{gathered}
$$

где $f_{i}(a t-x)$ - функция, описывающая прямые волны, распространяющиеся соответственно по участкам 1,2 и 3 в направлении оси $x ; \varphi_{i}(a t+x)$ - функции, описывающие обратные волны, распространяющиеся по участкам 1, 2 и 3 в противоположном направлении.

Перейдём к относительным величинам, характеризующим прямые и обратные волны $\tilde{f}^{\prime}(a t-x)=f^{\prime}(a t-x) a / V_{0} ; \tilde{\varphi}^{\prime}(a t+x)=\varphi^{\prime}(a t+x) a / V_{0}$, деформацию в сечении и его скорость $\tilde{\varepsilon}(x, t)=-\tilde{f}^{\prime}(a t-x)+\tilde{\varphi}^{\prime}(a t+x), \tilde{\nu}(x, t)=\nu(x, t) / V_{0}=\tilde{f}^{\prime}(a t-x)+$ $+\tilde{\varphi}^{\prime}(a t+x)$.

Осуществлено математическое моделирование продольного удара при длинах участков $l_{1}, l_{2}, l_{3}$ стержневой системы, указанных в таблице. Отношение площадей поперечных сечений каждого предыдущего участка к последующему, выражающему понижение продольной жёсткости поперечных сечений стержневой системы в направлении жёсткой преграды: $\tilde{A}=2$, $\tilde{A}=3$. Здесь $\tilde{A}=A_{1} / A_{2}=A_{2} / A_{3}$.

\begin{tabular}{c|c|c}
\hline $\begin{array}{c}\text { Длина } \\
\text { участка } 1\end{array}$ & $\begin{array}{c}\text { Длина } \\
\text { участка } 2\end{array}$ & $\begin{array}{c}\text { Длина } \\
\text { участка } 3\end{array}$ \\
\hline$l_{1}$ & $l_{2}$ & $l_{3}$ \\
$0,2 l$ & $0,2 l$ & $0,6 l$ \\
$0,2 l$ & $0,4 l$ & $0,4 l$ \\
$0,2 l$ & $0,6 l$ & $0,2 l$ \\
$0,4 l$ & $0,2 l$ & $0,4 l$ \\
$0,4 l$ & $0,4 l$ & $0,2 l$ \\
$0,6 l$ & $0,2 l$ & $0,2 l$ \\
\hline
\end{tabular}

4. Пример. В качестве примера с применением метода характеристик [1] построено поле волновых состояний при продольном ударе однородного и ступенчатого стержней о жёсткую преграду при $l_{1}=l_{2}=0,2 l, l_{3}=0,6 l, \tilde{A}=3$ (рис. 2). Области состояний $\mathrm{I}_{0}-\mathrm{I}_{16}, \mathrm{II}_{0}-\mathrm{II}_{17}, \mathrm{III}_{0}-\mathrm{III}_{26}$ с соответствующими значениями $\tilde{f}^{\prime}(a t-x)$, $\tilde{\varphi}^{\prime}(a t+x), \tilde{\varepsilon}(x, t), \tilde{\nu}(x, t)$ определяют параметры прямых и обратных волн деформаций, продольную деформацию и скорость поперечных сечений.

Зависимость величины максимальной относительной продольной деформации $\tilde{\varepsilon}_{\max }$ в опасных сечениях от длин участков $l_{1}, l_{2}, l_{3}$ и значения $\tilde{A}$ стержневой системы легко проиллюстрировать на графиках $\tilde{\varepsilon}_{\max }(l)$, представленных на рис. 3 (a, б). 


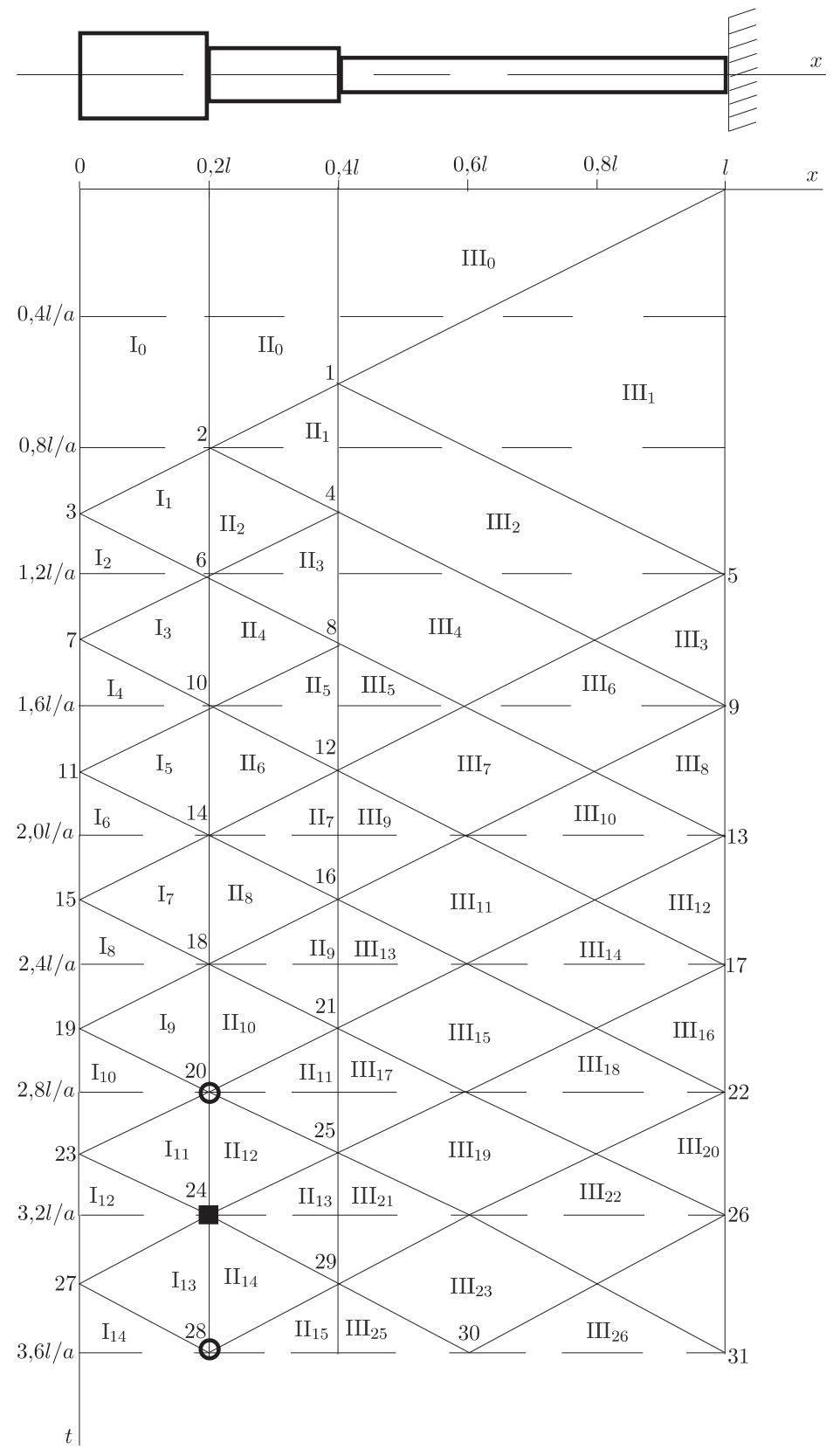

Рис. 2. Поле состояний при ударе стержневой системы о жёсткую преграду 

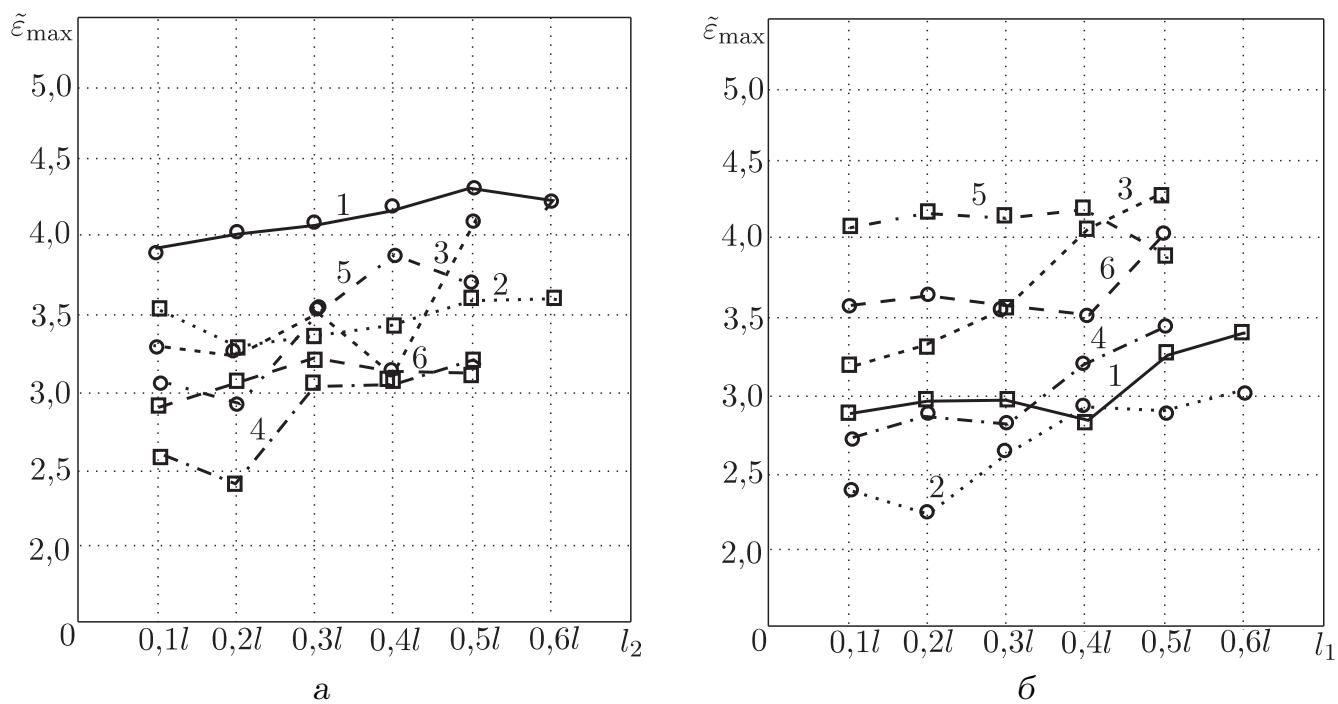

Рис. 3. Графики зависимости величины $\tilde{\varepsilon}_{\max }$ от соотношения длин и поперечных размеров однородных участков стержневой системы

График на рис. 3 , а представлен для $\tilde{\varepsilon}_{\max }$ в зависимости от длины начального участка ступенчатого стержня $l_{2}$ при постоянной длине $l_{1}$ однородного стержня. На рис. 3 , $\sigma$ представлен график для $\tilde{\varepsilon}_{\max }$ в зависимости от длины $l_{1}$ однородного стержня при постоянной длине $l_{2}$ начального участка ступенчатого стержня. Соответственно в обоих случаях будет изменяться длина однородного участка $l_{3}$, поскольку общая длина стержневой системы постоянна и равна $l$. На рис. 3 , а линия 1 выражает график зависимости $\tilde{\varepsilon}_{\max }\left(l_{2}\right)$ при $\tilde{A}=3$ и $l_{1}=0,2 l$, линия 2 выражает график зависимости $\tilde{\varepsilon}_{\max }\left(l_{2}\right)$ при $\tilde{A}=2$ при $l_{1}=0,2 l$, линия $3-$ при $\tilde{A}=3$ и $l_{1}=0,3 l$, линия $4-$ при $\tilde{A}=2$ и $l_{1}=0,3 l$, линия $5-$ при $\tilde{A}=3$ и $l_{1}=0,4 l$, линия $6-$ при $\tilde{A}=2$ и $l_{1}=0,4 l$. На рис. 3 , б представлен график зависимости $\tilde{\varepsilon}_{\max }\left(l_{1}\right)$ при значениях $l_{2}$, равных $0,2 l, 0,3 l, 0,4 l$, и $\tilde{A}$, равных 2 и 3 соответственно.

\section{Выводы.}

1. Исходя из анализа построенных графиков, можно сделать вывод, что величи-

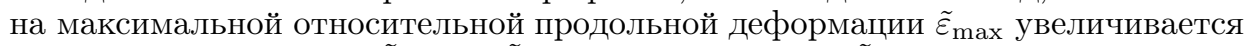
с ростом соотношения $\tilde{A}$. При $\tilde{A}=3 \tilde{\varepsilon}_{\max }=4,3$, при $\tilde{A}=2 \tilde{\varepsilon}_{\max }=4$.

2. Из анализа этих же графиков, необходимо отметить, что величина $\tilde{\varepsilon}_{\max }$ слабо зависит от соотношения длин участков стержневой системы $l_{1}, l_{2}, l_{3}$.

\section{БИБЛИОГРАФИЧЕСКИЙ СПИСОК}

1. Алимов О. Д., Манжсосов В. К., Еремъяни В.Э. Удар. Распространение волн деформаций в ударных системах. - М.: Наука, 1985. - 354 с.

2. Битюрин A. A., Манжосов B. К. Возникновение ненагруженного состояния в однородном стержне при соударении стержневой системы с жёсткой преградой // Изв. вузов. Машиностроение, 2006. - №11. - С. 27-32.

3. Битюрин A.A., Манжсосов В.К. Изменение деформации на участках стержневой системы после повторного удара в контактном сечении // Вестн. Улъянов. гос. техн. ун-та, 2007. - № 3. - С. 23-28.

4. Битюрин A. A., Манжосов B. K. Волновые процессы при продольном ударе стержня о ступенчатый стержень, соприкасающийся с жёсткой преградой // ПMM, 2009. - Т. 73, № 2. - C. 226-235. 
5. Битюрин A. A., Манжосов B. K. Математическое моделирование продольного удара неоднородной стержневой системы при увеличении продольной жесткости // $И з 8$. $C a-$ рат. ун-та. Нов. сер. Сер. Математика. Механика. Информатика, 2009. — Т. 9, № 2. 一 C. $66-73$.

6. Битюрин A. A., Манжосов B. K. Продольный удар неоднородного стержня о жёсткую преграду. - Ульяновск: УлГТУ, 2009. - 164 с.

Поступила в редакцию 04/IX/2009;

в окончательном варианте - 23/IX/2010.

MSC: 65M25

\section{LONGITUDINAL IMPACT ON RIGID BARRIER HOMOGENEOUS AND STEPPED BAR IS LOWERED LONGITUDINAL STIFFNESS}

\section{A. A. Bitjurin, V.K. Manzhosov}

Ulyanovsk State Technical University,

32, Severniy Venetz st., Ulyanovsk, 432027, Russia.

E-mail: Denjgy0706@yandex.ru

Mathematical modelling of longitudinal elastic central blow of non-uniform rod system about a rigid barrier is carried out, at not keeping communications by the analytical decision of the wave differential equation by a method of Dalambera. The rod system consists of a homogeneous core of constant cross-section section and the step nonuniform core having two homogeneous sites of various length and the area of crosssection sections. Ties with a rigid barrier and between cores are not witholding.

Key words: blow, deformation, mathematical modelling, speed.

Original article submitted 04/IX/2009; revision submitted 23/IX/2010.

Anatoly A. Bitjurin (Ph. D. (Techn.)), Associate Professor, Dept. of Theoretical \& Applied Mechanics. Vladmir K. Manzhosov (Dr. Sci. (Techn.)), Head of Dept., Dept. of Theoretical \& Applied Mechanics. 